CERN-PPE/97-018

11 February 1997

\title{
A Measurement of $\boldsymbol{R}_{b}$ using Mutually Exclusive Tags
}

\author{
The ALEPH collaboration
}

\begin{abstract}
A measurement of $R_{b}$ using five mutually exclusive hemisphere tags has been performed by ALEPH using the full LEP1 statistics. Three tags are designed to select the decay of the $Z^{0}$ to $b$ quarks, while the remaining two select $Z^{0}$ decays to $c$ and light quarks, and are used to measure the tagging efficiencies. The result, $R_{b}=0.2159 \pm 0.0009$ (stat) \pm 0.0011 (syst), is in agreement with the electroweak theory prediction of $0.2158 \pm 0.0003$.
\end{abstract}

(To be submitted to Physics Letters B) 


\section{The ALEPH Collaboration}

R. Barate, D. Buskulic, D. Decamp, P. Ghez, C. Goy, J.-P. Lees, A. Lucotte, M.-N. Minard, J.-Y. Nief, B. Pietrzyk

Laboratoire de Physique des Particules (LAPP), IN P $^{3}$-CNRS, 74019 Annecy-le-Vieux Cedex, France

M.P. Casado, M. Chmeissani, P. Comas, J.M. Crespo, M. Delfino, E. Fernandez, M. FernandezBosman, Ll. Garrido, ${ }^{15}$ A. Juste, M. Martinez, R. Miquel, Ll.M. Mir, S. Orteu, C. Padilla, I.C. Park, A. Pascual, J.A. Perlas, I. Riu, F. Sanchez, F. Teubert

Institut de Fisica d'Altes Energies, Universitat Autonoma de Barcelona, 08193 Bellaterra (Barcelona), Spain $^{7}$

A. Colaleo, D. Creanza, M. de Palma, G. Gelao, G. Iaselli, G. Maggi, M. Maggi, N. Marinelli, S. Nuzzo, A. Ranieri, G. Raso, F. Ruggieri, G. Selvaggi, L. Silvestris, P. Tempesta, A. Tricomi, ${ }^{3}$ G. Zito

Dipartimento di Fisica, INFN Sezione di Bari, 70126 Bari, Italy

X. Huang, J. Lin, Q. Ouyang, T. Wang, Y. Xie, R. Xu, S. Xue, J. Zhang, L. Zhang, W. Zhao

Institute of High-Energy Physics, Academia Sinica, Beijing, The People's Republic of China ${ }^{8}$

D. Abbaneo, R. Alemany, U. Becker, A.O. Bazarko, ${ }^{20}$ P. Bright-Thomas, M. Cattaneo, F. Cerutti, H. Drevermann, R.W. Forty, M. Frank, R. Hagelberg, J. Harvey, P. Janot, B. Jost, E. Kneringer, J. Knobloch, I. Lehraus, G. Lutters, P. Mato, A. Minten, L. Moneta, A. Pacheco, J.-F. Pusztaszeri, F. Ranjard, P. Rensing, ${ }^{12}$ G. Rizzo, L. Rolandi, D. Schlatter, M. Schmitt, O. Schneider, W. Tejessy, I.R. Tomalin, H. Wachsmuth, A. Wagner

European Laboratory for Particle Physics (CERN), 1211 Geneva 23, Switzerland

Z. Ajaltouni, A. Barrès, C. Boyer, A. Falvard, C. Ferdi, P. Gay, C . Guicheney, P. Henrard, J. Jousset, B. Michel, S. Monteil, J-C. Montret, D. Pallin, P. Perret, F. Podlyski, J. Proriol, P. Rosnet, J.M. Rossignol

Laboratoire de Physique Corpusculaire, Université Blaise Pascal, IN² $P^{3}$-CNRS, Clermont-Ferrand, 63177 Aubière, France

T. Fearnley, J.B. Hansen, J.D. Hansen, J.R. Hansen, P.H. Hansen, B.S. Nilsson, B. Rensch, A. Wäänänen

Niels Bohr Institute, 2100 Copenhagen, Denmark ${ }^{9}$

G. Daskalakis, A. Kyriakis, C. Markou, E. Simopoulou, I. Siotis, A. Vayaki

Nuclear Research Center Demokritos (NRCD), Athens, Greece

A. Blondel, G. Bonneaud, J.C. Brient, P. Bourdon, A. Rougé, M. Rumpf, A. Valassi, ${ }^{6}$ M. Verderi, H. Videau

Laboratoire de Physique Nucléaire et des Hautes Energies, Ecole Polytechnique, IN ${ }^{2} P^{3}$-CNRS, 91128 Palaiseau Cedex, France

D.J. Candlin, M.I. Parsons

Department of Physics, University of Edinburgh, Edinburgh EH9 3JZ, United Kingdom ${ }^{10}$

E. Focardi, G. Parrini, K. Zachariadou

Dipartimento di Fisica, Università di Firenze, INFN Sezione di Firenze, 50125 Firenze, Italy

M. Corden, C. Georgiopoulos, D.E. Jaffe

Supercomputer Computations Research Institute, Florida State University, Tallahassee, FL 323064052, USA ${ }^{13,14}$

A. Antonelli, G. Bencivenni, G. Bologna, ${ }^{4}$ F. Bossi, P. Campana, G. Capon, D. Casper, V. Chiarella, G. Felici, P. Laurelli, G. Mannocchi, ${ }^{5}$ F. Murtas, G.P. Murtas, L. Passalacqua, M. Pepe-Altarelli

Laboratori Nazionali dell'INFN (LNF-INFN), 00044 Frascati, Italy 
L. Curtis, S.J. Dorris, A.W. Halley, I.G. Knowles, J.G. Lynch, V. O'Shea, C. Raine, J.M. Scarr, K. Smith, P. Teixeira-Dias, A.S. Thompson, E. Thomson, F. Thomson, R.M. Turnbull

Department of Physics and Astronomy, University of Glasgow, Glasgow G12 8QQ,United Kingdom ${ }^{10}$

C. Geweniger, G. Graefe, P. Hanke, G. Hansper, V. Hepp, E.E. Kluge, A. Putzer, M. Schmidt, J. Sommer, K. Tittel, S. Werner, M. Wunsch

Institut für Hochenergiephysik, Universität Heidelberg, 69120 Heidelberg, Fed. Rep. of Germany ${ }^{16}$

R. Beuselinck, D.M. Binnie, W. Cameron, P.J. Dornan, M. Girone, S. Goodsir, E.B. Martin, A. Moutoussi, J. Nash, J.K. Sedgbeer, A.M. Stacey, M.D. Williams

Department of Physics, Imperial College, London SW7 2BZ, United Kingdom ${ }^{10}$

G. Dissertori, V.M. Ghete, P. Girtler, D. Kuhn, G. Rudolph

Institut für Experimentalphysik, Universität Innsbruck, 6020 Innsbruck, Austria ${ }^{18}$

A.P. Betteridge, C.K. Bowdery, P. Colrain, G. Crawford, A.J. Finch, F. Foster, G. Hughes, T. Sloan, M.I. Williams

Department of Physics, University of Lancaster, Lancaster LA1 4YB, United Kingdom ${ }^{10}$

A. Galla, I. Giehl, A.M. Greene, C. Hoffmann, K. Jakobs, K. Kleinknecht, G. Quast, B. Renk, E. Rohne, H.-G. Sander, P. van Gemmeren, C. Zeitnitz

Institut für Physik, Universität Mainz, 55099 Mainz, Fed. Rep. of Germany ${ }^{16}$

J.J. Aubert, C. Benchouk, A. Bonissent, G. Bujosa, D. Calvet, J. Carr, P. Coyle, C. Diaconu, F. Etienne, N. Konstantinidis, O. Leroy, F. Motsch, P. Payre, D. Rousseau, M. Talby, A. Sadouki, M. Thulasidas, K. Trabelsi

Centre de Physique des Particules, Faculté des Sciences de Luminy, $I N^{2} P^{3}$-CNRS, 13288 Marseille, France

M. Aleppo, F. Ragusa ${ }^{2}$

Dipartimento di Fisica, Università di Milano e INFN Sezione di Milano, 20133 Milano, Italy

R. Berlich, W. Blum, D. Brown, V. Büscher, H. Dietl, F. Dydak, ${ }^{2}$ G. Ganis, C. Gotzhein, H. Kroha, G. Lütjens, G. Lutz, W. Männer, H.-G. Moser, R. Richter, A. Rosado-Schlosser, S. Schael, R. Settles, H. Seywerd, R. St. Denis, H. Stenzel, W. Wiedenmann, G. Wolf

Max-Planck-Institut für Physik, Werner-Heisenberg-Institut, 80805 München, Fed. Rep. of Germany ${ }^{16}$

J. Boucrot, O. Callot, ${ }^{2}$ S. Chen, Y. Choi, ${ }^{21}$ A. Cordier, M. Davier, L. Duflot, J.-F. Grivaz, Ph. Heusse, A. Höcker, A. Jacholkowska, M. Jacquet, D.W. Kim ${ }^{24}$ F. Le Diberder, J. Lefrançois, A.-M. Lutz, I. Nikolic, M.-H. Schune, S. Simion, E. Tournefier, J.-J. Veillet, I. Videau, D. Zerwas

Laboratoire de l'Accélérateur Linéaire, Université de Paris-Sud, $I N^{2} P^{3}-C N R S, 91405$ Orsay Cedex, France

P. Azzurri, G. Bagliesi, G. Batignani, S. Bettarini, C. Bozzi, G. Calderini, M. Carpinelli, M.A. Ciocci, V. Ciulli, R. Dell'Orso, R. Fantechi, I. Ferrante, L. Foà, ${ }^{1}$ F. Forti, A. Giassi, M.A. Giorgi, A. Gregorio, F. Ligabue, A. Lusiani, P.S. Marrocchesi, A. Messineo, F. Palla, G. Sanguinetti, A. Sciabà, P. Spagnolo, J. Steinberger, R. Tenchini, G. Tonelli, ${ }^{19}$ C. Vannini, A. Venturi, P.G. Verdini

Dipartimento di Fisica dell'Università, INFN Sezione di Pisa, e Scuola Normale Superiore, 56010 Pisa, Italy

G.A. Blair, L.M. Bryant, J.T. Chambers, Y. Gao, M.G. Green, T. Medcalf, P. Perrodo, J.A. Strong, J.H. von Wimmersperg-Toeller

Department of Physics, Royal Holloway \& Bedford New College, University of London, Surrey TW20 OEX, United Kingdom ${ }^{10}$

D.R. Botterill, R.W. Clifft, T.R. Edgecock, S. Haywood, P. Maley, P.R. Norton, J.C. Thompson, A.E. Wright

Particle Physics Dept., Rutherford Appleton Laboratory, Chilton, Didcot, Oxon OX11 OQX, United Kingdom ${ }^{10}$ 
B. Bloch-Devaux, P. Colas, S. Emery, W. Kozanecki, E. Lançon, M.C. Lemaire, E. Locci, P. Perez, J. Rander, J.-F. Renardy, A. Roussarie, J.-P. Schuller, J. Schwindling, A. Trabelsi, B. Vallage

CEA, DAPNIA/Service de Physique des Particules, CE-Saclay, 91191 Gif-sur-Yvette Cedex, France ${ }^{17}$

S.N. Black, J.H. Dann, R.P. Johnson, H.Y. Kim, A.M. Litke, M.A. McNeil, G. Taylor

Institute for Particle Physics, University of California at Santa Cruz, Santa Cruz, CA 95064, USA ${ }^{22}$

C.N. Booth, R. Boswell, C.A.J. Brew, S. Cartwright, F. Combley, M.S. Kelly, M. Lehto, W.M. Newton, J. Reeve, L.F. Thompson

Department of Physics, University of Sheffield, Sheffield S3 7RH, United Kingdom ${ }^{10}$

A. Böhrer, S. Brandt, G. Cowan, C. Grupen, P. Saraiva, L. Smolik, F. Stephan

Fachbereich Physik, Universität Siegen, 57068 Siegen, Fed. Rep. of Germany ${ }^{16}$

M. Apollonio, L. Bosisio, R. Della Marina, G. Giannini, B. Gobbo, G. Musolino

Dipartimento di Fisica, Università di Trieste e INFN Sezione di Trieste, 34127 Trieste, Italy

J. Rothberg, S. Wasserbaech

Experimental Elementary Particle Physics, University of Washington, WA 98195 Seattle, U.S.A.

S.R. Armstrong, E. Charles, P. Elmer, D.P.S. Ferguson, Y.S. Gao, ${ }^{23}$ S. González, T.C. Greening, O.J. Hayes, H. Hu, S. Jin, P.A. McNamara III, J.M. Nachtman, J. Nielsen, W. Orejudos, Y.B. Pan, Y. Saadi, I.J. Scott, J. Walsh, Sau Lan Wu, X. Wu, J.M. Yamartino, G. Zobernig

Department of Physics, University of Wisconsin, Madison, WI 53706, USA ${ }^{11}$

\footnotetext{
${ }^{1}$ Now at CERN, 1211 Geneva 23, Switzerland.

${ }^{2}$ Also at CERN, 1211 Geneva 23, Switzerland.

${ }^{3}$ Also at Dipartimento di Fisica, INFN, Sezione di Catania, Catania, Italy.

${ }^{4}$ Also Istituto di Fisica Generale, Università di Torino, Torino, Italy.

${ }^{5}$ Also Istituto di Cosmo-Geofisica del C.N.R., Torino, Italy.

${ }^{6}$ Supported by the Commission of the European Communities, contract ERBCHBICT941234.

${ }^{7}$ Supported by CICYT, Spain.

${ }^{8}$ Supported by the National Science Foundation of China.

${ }^{9}$ Supported by the Danish Natural Science Research Council.

${ }^{10}$ Supported by the UK Particle Physics and Astronomy Research Council.

${ }^{11}$ Supported by the US Department of Energy, grant DE-FG0295-ER40896.

${ }^{12}$ Now at Dragon Systems, Newton, MA 02160, U.S.A.

${ }^{13}$ Supported by the US Department of Energy, contract DE-FG05-92ER40742.

${ }^{14}$ Supported by the US Department of Energy, contract DE-FC05-85ER250000.

${ }^{15}$ Permanent address: Universitat de Barcelona, 08208 Barcelona, Spain.

${ }^{16}$ Supported by the Bundesministerium für Bildung, Wissenschaft, Forschung und Technologie, Fed.

Rep. of Germany.

${ }^{17}$ Supported by the Direction des Sciences de la Matière, C.E.A.

${ }^{18}$ Supported by Fonds zur Förderung der wissenschaftlichen Forschung, Austria.

${ }^{19}$ Also at Istituto di Matematica e Fisica, Università di Sassari, Sassari, Italy.

${ }^{20}$ Now at Princeton University, Princeton, NJ 08544, U.S.A.

${ }^{21}$ Permanent address: Sung Kyun Kwan University, Suwon, Korea.

${ }^{22}$ Supported by the US Department of Energy, grant DE-FG03-92ER40689.

${ }^{23}$ Now at Harvard University, Cambridge, MA 02138, U.S.A.

${ }^{24}$ Permanent address: Kangnung National University, Kangnung, Korea.
} 


\section{Introduction}

The foregoing measurement [1] of $R_{b}$, the fraction of hadronic $Z^{0}$ decays to $b$ quarks, is based on hemisphere $b$ quark selection by means of one tag which utilises lifetime and mass information. In the present analysis, this lifetime-mass hemisphere tag is complemented by four other mutually exclusive tags, using event shape information as well as lifetime and leptons. Mutually exclusive here means that the tags are constructed such that a hemisphere will be tagged at most by one tag. In this way it has been possible to increase the statistical accuracy as well as to reduce the systematic uncertainty.

Three of the five tags are designed to $\operatorname{tag} b$ events, one is designed to select $c$ events, and one designed to select the combination of the three lighter quarks, $u, d$ and $s$, together. The $b$ tags include the lifetime-mass tag of [1], a tag that uses both lifetime and event shape information, and a tag based on the identification of leptons with large momenta and transverse momenta. The lifetime-mass tag has the highest purity and the largest impact on the analysis.

The five mutually exclusive hemisphere tags result in 20 statistically independent measurements: 5 singly tagged fractions, 5 doubly tagged with the same tag, and 10 doubly tagged with different tags. These are used in the present analysis to determine 14 quantities: $R_{b}$ and 13 of the 15 efficiencies of the 5 tags for $b$, for $c$ and for the combination of $u, d, s$ flavours. The two background efficiencies of the lifetime-mass tag cannot be determined experimentally with success. There remain six constraints, which serve as a check on the analysis.

Monte Carlo simulation is used to calculate the two lifetime-mass tag background efficiencies and the correlations in the tagging of the two hemispheres in the same event. These contribute the dominant systematic uncertainties of the analysis.

\section{The Method}

Events are divided into hemispheres by the plane perpendicular to the thrust axis.

The fraction of tagged hemispheres $f_{s}^{I}$ with $\operatorname{tag} I$ is

$$
f_{s}^{I}=R_{b} \epsilon_{b}^{I}+R_{c} \epsilon_{c}^{I}+\left(1-R_{b}-R_{c}\right) \epsilon_{x}^{I}
$$

where $R_{b}$ and $R_{c}$ are the $b$ and $c$ branching fractions of hadronic $Z^{0}$ decays and $\epsilon_{a}^{I}$ are the hemisphere tagging efficiencies for flavour $a$ using tag $I . \epsilon_{x}$ is the average efficiency for $u, d$ and $s$ flavours. For five tags, there are $3 \times 5=15$ efficiencies.

The fraction of doubly tagged events $f_{d}^{I, J}$ with tags $I$ and $J$ is

$$
\begin{aligned}
f_{d}^{I, J}= & {\left[R_{b} \epsilon_{b}^{I} \epsilon_{b}^{J}\left(1+\rho_{b}^{I, J}\right)+R_{c} \epsilon_{c}^{I} \epsilon_{c}^{J}\left(1+\rho_{c}^{I, J}\right)+\right.} \\
& \left.\left(1-R_{b}-R_{c}\right) \epsilon_{x}^{I} \epsilon_{x}^{J}\left(1+\rho_{x}^{I, J}\right)\right]\left(2-\delta_{I, J}\right)
\end{aligned}
$$

where $\rho_{a}^{I, J}$ are the hemisphere-hemisphere efficiency correlations for flavour $a$ and tags $I$ and $J$. The effects on the correlations due to the small differences in efficiencies between $u, d$ and $s$ flavours are taken into account in the calculation of $\rho_{x}^{I, J}$. There are $3 \times 15=45$ correlations. 
The 20 independent measured quantities consist of the 5 numbers of singly tagged events, $N_{s}^{I}$

$$
N_{s}^{I}=N\left(2 f_{s}^{I}-\sum_{K=1}^{5} f_{d}^{I, K} \cdot\left(1+\delta_{I, K}\right)\right)
$$

where $N$ is the total number of events, and the 15 numbers of doubly tagged events $N_{d}^{I, J}$

$$
N_{d}^{I, J}=N f_{d}^{I, J}
$$

These 20 measurements are described by 62 unknown parameters: $R_{b}, R_{c}$, the 15 efficiencies of the five tags for the three flavours, and the 45 correlations of the 15 pairs of tags for the three flavours. In the following analysis $R_{b}$ and 13 efficiencies are fitted to the data. The remaining two efficiencies of the lifetime-mass tag, $\epsilon_{c}^{Q}$ and $\epsilon_{x}^{Q}$, and the 45 correlations are calculated using Monte Carlo simulation. The systematic error reflects the uncertainties in these calculations. The result will be given as a function of the difference between $R_{c}$ and its value of 0.172 in the electroweak theory.

\section{Event selection}

The data used for the analysis were obtained on and near the $Z^{0}$ resonance in the ALEPH detector [2] during the period 1992 to 1995, since the introduction of a double sided microstrip vertex detector, with strip readout in $r-\phi$ and $z$.

Events are selected as in [1] except for the following requirements:

a) $\left|\cos \theta_{\text {THRUST }}\right|<0.65$, where $\theta_{\text {THRUST }}$ is the angle between the beam and the thrust axes.

b) $y_{3}<0.2$, where $y_{3}$ is the value of $y_{\text {cut }}$ that sets the transition from 2 to 3 jets using the JADE algorithm [3]. This cut eliminates the $3 \%$ of events with the largest gluon radiation, for which the correspondence between data and Monte Carlo is poorer.

There remain 2,057,618 events. The Monte Carlo simulation is used to determine a selection bias in favour of $b$ quarks relative to the lighter quarks of $0.1 \pm 0.1 \%$, where the error is dominated by statistics, and a contamination from tau events of $0.30 \pm 0.01 \%$, where the error is dominated by systematics.

\section{The Five Hemisphere Tags}

The tags are designed in a pragmatic attempt to isolate the desired quark flavour with high efficiency and purity while keeping the hemisphere-hemisphere correlations small. The latter is accomplished by deriving the tags from hemisphere quantities exclusively. In particular, the primary $\left(Z^{0}\right.$ decay) vertex is reconstructed independently in the two hemispheres, as described in [1].

The five tags are constructed from the following eight derived hemisphere quantities, of which the first two are described in greater detail in [1]: 
1. $\mathcal{P}_{H}$, the confidence level that all of the hemisphere tracks originate from the primary hemisphere vertex.

2. $\mu_{H}$, the variable related to the invariant mass of the tracks inconsistent with originating from the primary vertex. Tracks in a hemisphere are ordered inversely to their probability $\mathcal{P}_{T}$ to originate from the primary vertex. Tracks are combined, in this order, until the invariant mass of the combination exceeds $1.8 \mathrm{GeV} / \mathrm{c}^{2}$. The quantity $\mu_{H}$ is the $\mathcal{P}_{T}$ of the last track added.

3. $\mathcal{N}_{B}$, the output of a neural network [4] trained to select $b$ quark hemispheres. The input quantities to the neural network are 25 event shape quantities, of which none depend explicitly on $b$ lifetime effects. These inputs are listed in the Appendix.

4. $p$, the momentum of an identified electron or muon. Lepton identification is described in [5]: electrons are primarily identified by their characteristic shower development in the calorimeter, and muons are primarily identified by their penetration pattern. If more than one lepton is found in a hemisphere the highest momentum lepton is used.

5. $p_{\perp}$, the transverse momentum of the lepton with respect to the direction of its jet after removing the lepton from the jet.

6. $\mathcal{N}_{C}$, the output of a neural network trained to select $c$ quark hemispheres. The neural net inputs are one lifetime and 19 event shape quantities, which are given in the Appendix.

7. $\mathcal{P}_{+}$, a variable used together with $\mathcal{P}_{-}$, to select $c$ quark hemispheres. Hemisphere tracks are divided into two groups on the basis of rapidity with respect to their associated jet axes greater or less than 5.1, chosen so that $b$ hemispheres find equal numbers of tracks in the two groups. $\mathcal{P}_{+}$is the confidence level that the tracks in the higher rapidity group originate from the primary vertex.

8. $\mathcal{P}_{-}$, the confidence level that the tracks in the lower rapidity group originate from the primary vertex.

Distributions of these eight variables are shown in Figures 1 and 2, where the Monte Carlo distributions are given for $b, c$ and $x$, together with a comparison of the flavourcombined Monte Carlo distributions with the data. Disagreements exist between the data and the Monte Carlo simulation, particularly for $\mathcal{N}_{C}$. The analysis is insensitive to such disagreements as they affect the efficiencies. The inadequacies of the Monte Carlo simulation as concerns the correlations are discussed in Section 6.3.

The definitions of the five tags in terms of the eight variables are given below. In order to satisfy the exclusive tag requirement each tag is given a priority, and if a hemisphere satisfies more than one tag, it is assigned to the tag with the highest priority. The lifetime-mass tag variable is given by

$$
\mathcal{B}_{l m}=-\left(0.7 \log _{10} \mu_{H}+0.3 \log _{10} \mathcal{P}_{H}\right)
$$



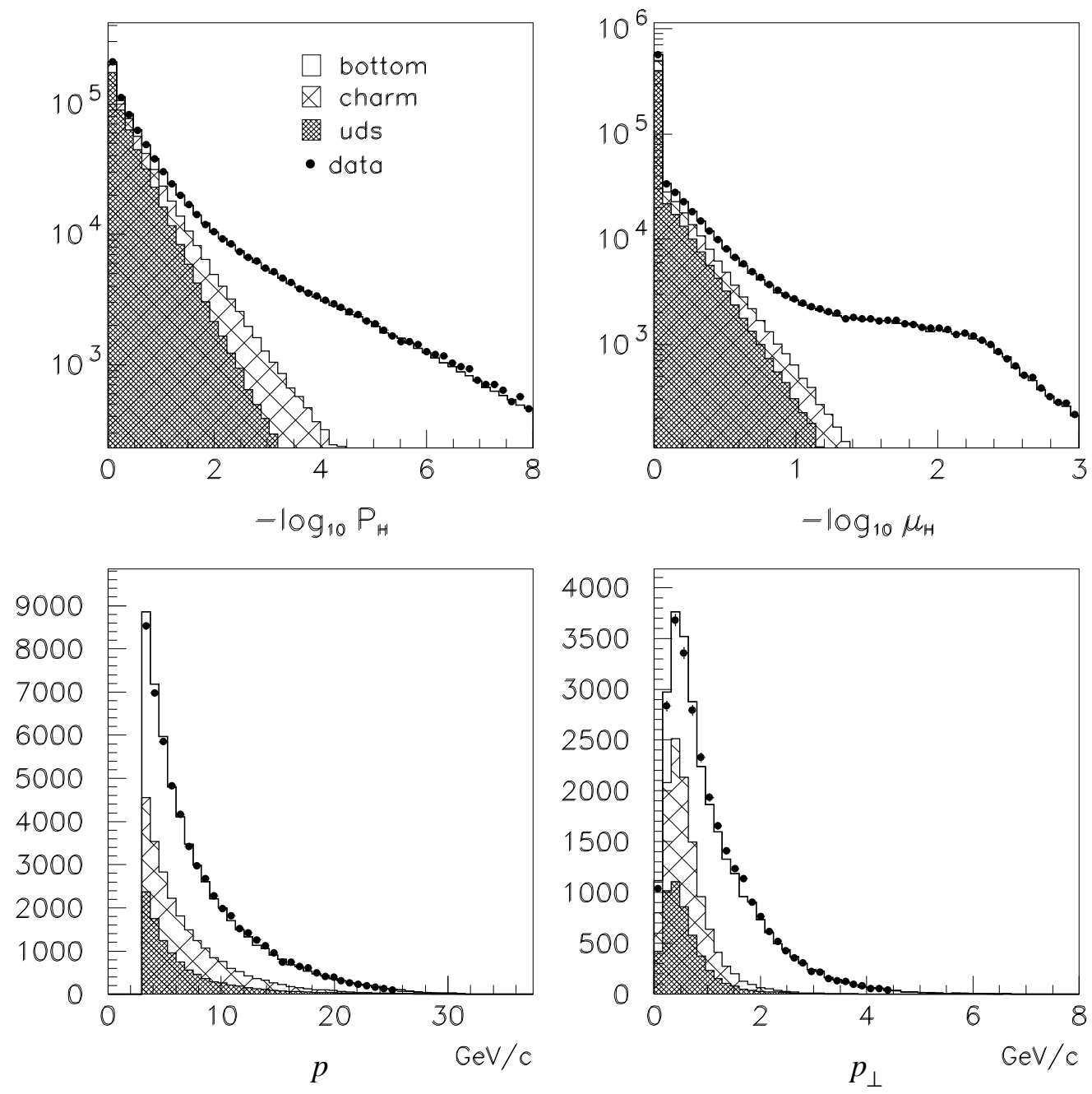

Figure 1: Comparison of data (points) and Monte Carlo simulation (histogram) for tagging variables $\mathcal{P}_{H}, \mu_{H}, p$, and $p_{\perp}$. The vertical axis is the number of hemispheres in the data per bin. The $p$, and $p_{\perp}$ spectra are not reweighted using the latest measurements of the semileptonic branching ratios. Quark flavour contributions in the simulation are indicated by the shaded regions. 

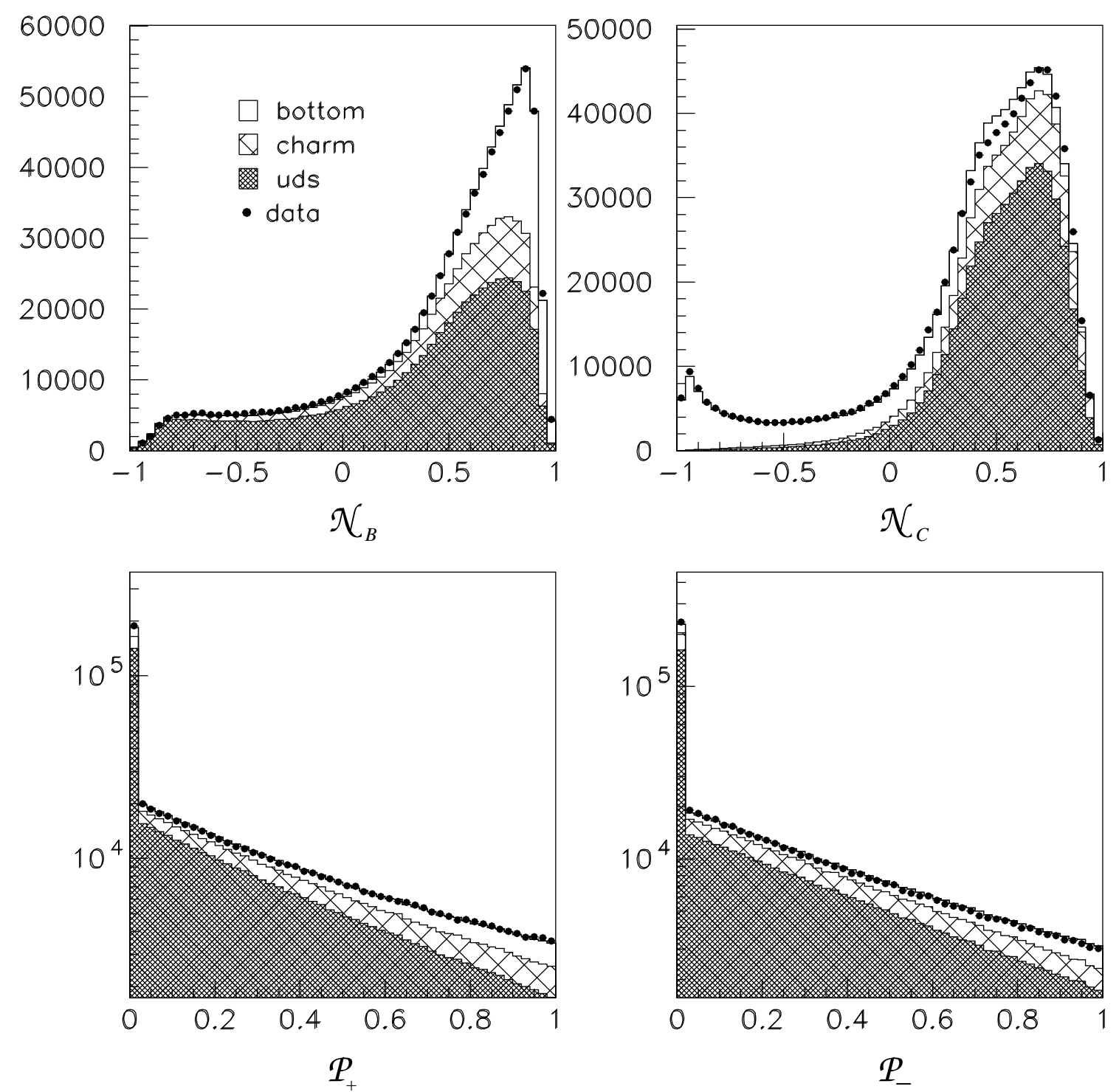

Figure 2: Comparison of data (points) and Monte Carlo simulation (histogram) for tagging variables $\mathcal{N}_{B}, \mathcal{N}_{C}, \mathcal{P}_{+}$, and $\mathcal{P}_{-}$. The vertical axis is the number of hemispheres in the data per bin. Quark flavour contributions in the simulation are indicated by the shaded regions. 
and the five tags are:

$$
\begin{array}{ccc}
\text { Q tag: } & \mathcal{B}_{l m}>2.2 & \text { Priority } 1 \\
\text { S tag: } & 0.85<\mathcal{B}_{l m}<2.2 \text { and } \mathcal{N}_{B}>\left(1.05-0.2 \mathcal{B}_{l m}\right) & \text { Priority } 2 \\
\text { L tag: } & p>3 \mathrm{GeV} / \mathrm{c} \text { and } p_{\perp}>1.4 \mathrm{GeV} / \mathrm{c} & \text { Priority } 5 \\
\text { C tag: } & \mathcal{P}_{-}>0.07 \text { and } 0.0003<\mathcal{P}_{+}<0.3 & \\
& \text { and } \mathcal{B}_{l m}<1.5 \text { and } \mathcal{N}_{C}>0.68 & \text { Priority } 3 \\
\text { X tag: } & \log _{10} \mathcal{P}_{H}>-0.25 \text { and } \mathcal{N}_{B}<0 & \text { Priority } 4
\end{array}
$$

The Monte Carlo expectations for the 15 efficiencies are given in Table 1 . These are a measure of the performance of the tags. In the $R_{b}$ determination only the charm and light quark efficiencies of the $\mathrm{Q}$ tag are taken from Monte Carlo simulation. The tighter cut on the $\mathrm{Q}$ tag with respect to the foregoing paper [1] results in a reduction in the background charm and $u, d, s$ efficiencies of nearly a factor of two.

\begin{tabular}{lrrr}
\hline tag & $\epsilon_{u d s}$ & $\epsilon_{c}$ & $\epsilon_{b}$ \\
\hline \hline $\mathrm{Q}$ & 0.00043 & 0.00216 & 0.1957 \\
$\mathrm{~S}$ & 0.00204 & 0.01402 & 0.1759 \\
$\mathrm{~L}$ & 0.00158 & 0.00694 & 0.0425 \\
$\mathrm{C}$ & 0.07927 & 0.16193 & 0.0260 \\
$\mathrm{X}$ & 0.11686 & 0.03962 & 0.0022 \\
\hline
\end{tabular}

Table 1: Monte Carlo results for the tagging efficiencies.

The Monte Carlo simulation is used to determine the hemisphere-hemisphere correlations for all tags. The impact on $R_{b}$ of a particular correlation is given by

$$
\frac{\Delta R_{b}}{R_{b} \Delta \rho_{a}^{I, J}},
$$

that is, the relative uncertainty in $R_{b}$ is the impact times the uncertainty in the correlation. The impacts vary a great deal, so that only about 10 of the 45 correlations are significant to the analysis. The Monte Carlo expectations for the dominant correlations and their impacts are given in Table 2. For comparison, in the single tag analysis [1] the impact of the single correlation considered is unity and the impacts of the other two correlations are negligible.

\section{Results}

The basic experimental result consists of 20 measurements: the numbers of singly and doubly tagged events. These are given in Table 3 . The fit of $R_{b}$ and 13 of the 15 efficiencies to these 20 data points gives the result

$$
R_{b}=0.21585 \pm 0.00087
$$




\begin{tabular}{crr}
\hline correlation & \multicolumn{1}{c}{ value } & impact \\
\hline \hline$\rho_{b}^{Q, Q}$ & $0.0274 \pm 0.0033$ & 0.450 \\
$\rho_{b}^{Q, S}$ & $-0.0022 \pm 0.0025$ & 0.420 \\
$\rho_{b}^{Q, L}$ & $-0.0176 \pm 0.0050$ & 0.084 \\
$\rho_{b}^{Q, C}$ & $0.0246 \pm 0.0072$ & -0.084 \\
$\rho_{x}^{X, C}$ & $0.0964 \pm 0.0049$ & -0.070 \\
$\rho_{b}^{S, C}$ & $0.0226 \pm 0.0076$ & 0.064 \\
$\rho_{x}^{C, C}$ & $0.0718 \pm 0.0085$ & -0.045 \\
$\rho_{c}^{C, C}$ & $0.0872 \pm 0.0075$ & -0.041 \\
$\rho_{b}^{S, S}$ & $-0.0060 \pm 0.0036$ & 0.026 \\
$\rho_{c}^{S, C}$ & $-0.0085 \pm 0.0179$ & 0.021 \\
$\rho_{x}^{X, X}$ & $0.1335 \pm 0.0056$ & -0.016 \\
$\rho_{c}^{X, C}$ & $0.0984 \pm 0.0120$ & -0.012 \\
$\rho_{b}^{L, C}$ & $0.0076 \pm 0.0168$ & 0.010 \\
\hline
\end{tabular}

Table 2: Monte Carlo results for the dominant correlations with statistical errors and their impacts on $R_{b}$.

with a $\chi^{2}$ of 8.1 for the 6 degrees of freedom. The error is the statistical error. This result has been corrected for the event selection bias and tau contamination discussed in section 3. In addition, a correction of +0.0003 has been applied to remove the contribution of the photon propagator.

\begin{tabular}{rr}
\hline$N_{Q}=96504$ & $N_{Q Q}=16715$ \\
$N_{S}=100266$ & $N_{S S}=13980$ \\
$N_{L}=33973$ & $N_{L L}=1097$ \\
$N_{X}=243002$ & $N_{X X}=18947$ \\
$N_{C}=265994$ & $N_{C C}=20123$ \\
$N_{Q S}=30138$ & $N_{Q L}=8098$ \\
$N_{Q X}=504$ & $N_{Q C}=4331$ \\
$N_{S L}=7534$ & $N_{S X}=1480$ \\
$N_{S C}=5838$ & $N_{L X}=1079$ \\
$N_{L C}=2570$ & $N_{X C}=32293$ \\
\hline
\end{tabular}

Table 3: Measured numbers of singly and doubly tagged events.

The fitted efficiencies can be compared with the Monte Carlo simulation predictions and are given together in Table 4 .

\section{Systematic errors}

The systematic errors arise only through the quantities calculated using Monte Carlo simulation: the uncertainty due to event selection, $\Delta R_{b}=0.00017$, the correlations 


\begin{tabular}{ccc}
\hline efficiency & $\begin{array}{c}\text { simulation } \\
\text { prediction }\end{array}$ & fit result \\
\hline \hline$\epsilon_{b}^{Q}$ & 0.1957 & $0.1922 \pm 0.0008$ \\
$\epsilon_{b}^{S}$ & 0.1759 & $0.1769 \pm 0.0006$ \\
$\epsilon_{c}^{S}$ & 0.0140 & $0.0128 \pm 0.0012$ \\
$\epsilon_{x}^{S}$ & 0.0020 & $0.0028 \pm 0.0002$ \\
$\epsilon_{b}^{L}$ & 0.0425 & $0.0486 \pm 0.0003$ \\
$\epsilon_{c}^{L}$ & 0.0069 & $0.0083 \pm 0.0007$ \\
$\epsilon_{x}^{L}$ & 0.0016 & $0.0031 \pm 0.0002$ \\
$\epsilon_{b}^{C}$ & 0.0260 & $0.0233 \pm 0.0004$ \\
$\epsilon_{c}^{C}$ & 0.1619 & $0.1592 \pm 0.0020$ \\
$\epsilon_{x}^{C}$ & 0.0793 & $0.0866 \pm 0.0005$ \\
$\epsilon_{b}^{X}$ & 0.0022 & $0.0021 \pm 0.0001$ \\
$\epsilon_{c}^{X}$ & 0.0396 & $0.0418 \pm 0.0005$ \\
$\epsilon_{x}^{X}$ & 0.1169 & $0.1129 \pm 0.0006$ \\
\hline
\end{tabular}

Table 4: Tagging efficiencies as predicted by Monte Carlo simulation and the fitted efficiencies with statistical errors. For a complete comparison of the fit results with the simulation predictions an estimate of the systematic error must be included.

and the two $\mathrm{Q}$ tag background efficiencies. The impacts on $R_{b}$ of the correlations are listed in Table 2, and the impacts of the two Q tag background efficiencies are given by

$$
\frac{\Delta R_{b}}{R_{b}} \frac{\epsilon_{b}^{Q}}{\Delta \epsilon_{c}^{Q}}=-1.5, \quad \frac{\Delta R_{b}}{R_{b}} \frac{\epsilon_{b}^{Q}}{\Delta \epsilon_{x}^{Q}}=-5.4 .
$$

This section describes uncertainties entering via these Monte Carlo quantities. The total uncertainty due to the limited Monte Carlo statistics is $\Delta R_{b}=0.00047$.

The task of estimating the systematic errors is essentially the same as described in [1], with the following observations:

1. The two $\mathrm{Q}$ tag background efficiencies enter with the same impacts in the two analyses. However, because of the harder cut $\left(\mathcal{B}_{l m}>2.2\right.$ compared with $\left.\mathcal{B}_{l m}>1.9\right)$ the background efficiencies relative to the $b$ efficiency are reduced by almost a factor of two, with a reduction in systematic error.

2. The impact of $\rho_{b}^{Q, Q}$ is reduced by more than a factor of two, but other correlations also contribute substantially to the error in $R_{b}$.

\subsection{Detector simulation uncertainty}

Monte Carlo predictions for $\epsilon_{c}^{Q}$ and $\epsilon_{x}^{Q}$ depend on the assumed impact parameter resolution and efficiency for vertex detector hits to be associated to tracks. As described in [1], the Monte Carlo simulation is corrected to achieve better agreement with data in these tracking quantities, and a systematic uncertainty is assigned to the correction. The resulting uncertainties in $\epsilon_{c}^{Q}$ and $\epsilon_{x}^{Q}$ are given in Table 5. 


\begin{tabular}{|l|c|c|c|}
\hline & $\Delta \epsilon_{c}^{Q}$ & $\Delta \epsilon_{x}^{Q}$ & $\Delta R_{b}$ \\
\hline$\theta$ dependence of $S$ resolution & 0.00002 & $<0.00001$ & 0.00003 \\
$p$ dependence of $S$ resolution & 0.00002 & 0.00001 & 0.00009 \\
$\theta$ dependence of tracking efficiency & 0.00001 & $<0.00001$ & 0.00002 \\
$p$ dependence of tracking efficiency & $<0.00001$ & $<0.00001$ & $<0.00001$ \\
Remaining inaccuracy of $S$ resolution & 0.00003 & $<0.00001$ & 0.00005 \\
Track correlation in $S$ resolution & 0.00008 & 0.00005 & 0.00045 \\
\hline Total uncertainty & 0.00009 & 0.00005 & 0.00046 \\
\hline
\end{tabular}

Table 5: Uncertainties in $\mathrm{Q}$ tag efficiencies and in $R_{b}$ due to uncertainties in detector simulation from the resolution on the impact parameter significance, $S[1]$, and tracking efficiency.

\subsection{Systematics from $b$ and $c$ physics uncertainties}

Uncertainties in physical parameters that enter into the simulation result in uncertainties in $\epsilon_{c}^{Q}, \epsilon_{x}^{Q}$ and the correlations.

These are calculated by varying the physics inputs to the Monte Carlo simulation within their allowed experimental ranges [6]. The procedure is described in reference $[1]$.

Table 6 reports the errors in $R_{b}$ due to these physical parameter variations. The overall error resulting from these uncertainties is $\Delta R_{b}=0.00084$.

\subsection{Correlation errors}

Correlation errors are assigned following the same basic procedure as in [1]. Since the correlation now concerns events in which the two hemispheres may be tagged by different tags, I and J, the contribution to the correlation $\rho_{f}^{I, J}$ is

$$
\rho_{f}^{I, J}(\mathrm{v})=\frac{\int f_{f}(\mathrm{v})\left[\epsilon_{f}^{I, \text { same }}(\mathrm{v}) \epsilon_{f}^{J, o p p o}(\mathrm{v})+\epsilon_{f}^{J, \text { same }}(\mathrm{v}) \epsilon_{f}^{I, \text { oppo }}(\mathrm{v})\right] d \mathrm{v}}{2\left\langle\epsilon_{f}^{I}\right\rangle\left\langle\epsilon_{f}^{J}\right\rangle}-1
$$

where $f_{f}(\mathrm{v})$ is the fractional event distribution in variable $\mathrm{v}$ for flavour $f, \epsilon_{f}^{I \text {,same }}(\mathrm{v})$ is the corresponding efficiency for the $\operatorname{tag} I$ for the hemisphere in which $\mathrm{v}$ is measured, and $\epsilon_{f}^{J, o p p o}(\mathrm{v})$ is the efficiency for the tag $J$ on the side opposite to the one in which $\mathrm{v}$ is measured.

The errors are assigned as the differences between data and Monte Carlo multiplied by the relevant impacts on $R_{b}$, summed linearly for all correlations for a given variable, and in quadrature for the four chosen variables: jet momentum, $y_{3}, \cos \theta_{\text {THRUST }}$ and $\phi_{\text {THRUST. }}$ If, instead, the sum were made quadratically for each variable, taking the larger of the difference between data and Monte Carlo or its error, the resulting systematic error would be slightly smaller.

Comparison of data and Monte Carlo simulation for a particular flavour requires the isolation of this flavour in the data. For the flavour isolation the same procedure 


\begin{tabular}{|c|c|}
\hline Source & $\overline{\Delta R_{b}}$ \\
\hline Both $b$ in the same hemisphere $: 2.2 \pm 0.7 \%$ & $\mp 0.00011$ \\
\hline $\mathrm{g} \rightarrow \mathrm{c} \overline{\mathrm{c}}:(2.38 \pm 0.48) \%$ per event & $\mp 0.00043$ \\
\hline Ratio $\mathrm{g} \rightarrow \mathrm{b} \bar{b} / \mathrm{g} \rightarrow \mathrm{c} \overline{\mathrm{c}}: 0.13 \pm 0.04$ & $\mp 0.00054$ \\
\hline$\left\langle x_{E}(\mathrm{~g})\right\rangle=0.76 \pm 0.03$ & $\mp 0.00010$ \\
\hline$B$ fragmentation : $0.702 \pm 0.008$ & $\mp 0.00010$ \\
\hline$B_{s}$ fraction : $0.112 \pm 0.019$ & $\mp 0.00004$ \\
\hline$\Lambda_{b}$ fraction $: 0.132 \pm 0.041$ & $\mp 0.00007$ \\
\hline$B^{+}$lifetime $: 1.62 \pm 0.06 \mathrm{ps}$ & $\mp 0.00005$ \\
\hline$B^{0}$ lifetime $: 1.56 \pm 0.06 \mathrm{ps}$ & $\mp 0.00005$ \\
\hline$B_{s}$ lifetime $: 1.61 \pm 0.10 \mathrm{ps}$ & $\mp 0.00003$ \\
\hline$\Lambda_{b}$ lifetime $: 1.14 \pm 0.08 \mathrm{ps}$ & $\mp 0.00003$ \\
\hline$B$ charged multiplicity : $5.73 \pm 0.35$ & $\mp 0.00017$ \\
\hline Charm fragmentation : $0.484 \pm 0.008$ & \pm 0.00011 \\
\hline$D^{+}$lifetime $: 1.057 \pm 0.015 \mathrm{ps}$ & $\mp 0.00001$ \\
\hline$D^{0}$ lifetime $: 0.415 \pm 0.004 \mathrm{ps}$ & $\mp 0.00001$ \\
\hline$D_{s}$ lifetime $: 0.467 \pm 0.017 \mathrm{ps}$ & $\mp 0.00004$ \\
\hline$\Lambda_{c}$ lifetime $: 0.206 \pm 0.012 \mathrm{ps}$ & $\mp 0.00001$ \\
\hline $\mathcal{B}\left(D^{+} \rightarrow K_{S}\right): 0.295 \pm 0.035$ & \pm 0.00009 \\
\hline $\mathcal{B}\left(D^{0} \rightarrow K_{S}\right): 0.210 \pm 0.025$ & \pm 0.00009 \\
\hline $\mathcal{B}\left(D_{s} \rightarrow K_{S}\right): 0.195 \pm 0.140$ & \pm 0.00012 \\
\hline$D^{+}$fraction $: 0.233 \pm 0.028$ & $\mp 0.00012$ \\
\hline$\Lambda_{c}$ fraction $: 0.065 \pm 0.029$ & \pm 0.00007 \\
\hline$D_{s}$ fraction : $0.102 \pm 0.037$ & $\mp 0.00003$ \\
\hline Charm charged multiplicity & $\mp 0.00025$ \\
\hline Charm neutral multiplicity & $\mp 0.00016$ \\
\hline $\mathrm{V}^{0}$ rate $( \pm 10 \%)$ and eff. $( \pm 20 \%)$ & $\mp 0.00004$ \\
\hline Total & 0.00084 \\
\hline
\end{tabular}

Table 6: Systematic errors in $R_{b}$ due to uncertainties in charm and bottom physics.

is used as in [1], resulting in two values for the data for the two different subtraction schemes (method 1 and method 2) which could be compared and whose average is used in the error assignment.

The isolation of the $b$ flavour uses the requirement $\mathcal{B}_{l m}>0.3$ for both hemispheres, with the resulting efficiencies $0.64,0.18$ and 0.03 for $b, c$ and $u, d, s$ flavours respectively.

For the isolation of the $u, d, s$ flavours the requirements $-\log _{10} \mathcal{P}_{H}<0.6$ and $\mathcal{P}_{+}>0.13$ are imposed on both hemispheres, with efficiencies $0.024,0.22$ and 0.54 for $b, c$ and $u, d, s$ flavours respectively.

No initial event selection requirement proved useful for purifying $c$ flavour. As a consequence, the flavour isolation algorithm in which the unwanted flavours in the data are subtracted on the basis of the Monte Carlo (method 1) suffers from very large statistical errors and cannot be used. The errors for $c$ flavour correlations are therefore assigned on the basis of the difference between the other method (method 2) and Monte 
Carlo.

Table 7 presents the results for the thirteen correlations with impact greater than 0.01. For each correlation, the overall value and impact are listed together with the contributions from the four variables studied. For each variable, the difference in background subtraction $\Delta D$ and the differences between data and Monte Carlo simulation are shown. The resultant uncertainty is $\Delta R_{b}=0.00027$. The error in $R_{b}$ due to the uncertainties in the remaining 32 correlations of very small impact is assigned by assuming $\pm 20 \%$ uncertainties in each of these. The resultant error in $R_{b}$ of \pm 0.00005 , combined with the errors due to the 13 larger impact correlations is finally $\Delta R_{b}=0.00027$.

\subsection{Systematic Error Summary}

The systematic errors are summarised below:

$$
\begin{aligned}
& \Delta R_{b}= \pm 0.00047 \text { Monte Carlo statistics } \\
& \pm 0.00017 \text { Event selection } \\
& \pm 0.00084 \text { Physics uncertainty } \\
& \pm 0.00046 \text { Tracking uncertainty } \\
& \pm 0.00027 \text { Hemisphere correlations uncertainty }
\end{aligned}
$$

\section{Discussion of the Result}

The systematic uncertainties evaluated in the previous section, added in quadrature, give a total systematic error of 0.00110 in $R_{b}$. The result presented in Section 5 becomes:

$$
R_{b}=0.2159 \pm 0.0009(\text { stat }) \pm 0.0011(\text { syst })-0.019 \times\left(R_{c}-0.172\right)
$$

where the explicit dependence on $R_{c}$ is given.

Figure 3 shows the stability of the result as a function of the cut on $\mathcal{N}_{C}$ of the $\mathrm{C}$ tag as well as a function of the $\mathcal{N}_{B}$ cut of the $\mathrm{S}$ tag. Figure 4 shows the stability of the result with respect to the $\mathrm{Q}$ tag cut, together with the contributions to the error.

The present analysis relies heavily on the lifetime-mass tag which is the basis of the preceding paper [1]. The result of this single tag analysis is

$$
R_{b}=0.2167 \pm 0.0011(\text { stat }) \pm 0.0013(\text { syst })-0.037 \times\left(R_{c}-0.172\right) .
$$

That result is highly correlated to the present one, both in the data used and in the systematic errors, so the two cannot be used independently. They are statistically consistent. The result presented here has the smaller error, and is therefore taken as the final result.

The result is in good agreement with the current expectation of Electroweak theory [7] as predicted for a top mass of $175 \pm 6 \mathrm{GeV} / \mathrm{c}^{2}$ [8]

$$
R_{b}=0.2158 \pm 0.0003 \quad \text { (Electroweak expectation). }
$$




\begin{tabular}{|c|c|c|c|c|c|c|c|c|c|c|c|c|c|c|c|c|c|c|}
\hline 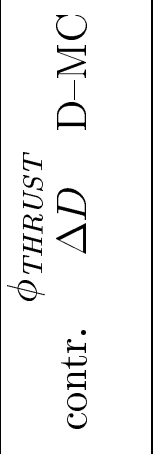 & & 8. & $\begin{array}{c}1 \\
8 \\
8 \\
0 \\
1\end{array}$ & ठ্ঠ & 蜜 & ஓ & 官 & 急 & & $\mid$\begin{tabular}{c}
$\infty$ \\
$\mathscr{8}$ \\
\hdashline \\
$\vdots$ \\
\end{tabular} & $\begin{array}{l}1 \\
8 \\
8 \\
0 \\
1 \\
8 \\
8 \\
8 \\
8\end{array}$ & $\begin{array}{l}5 \\
8 \\
8 \\
1 \\
8 \\
8 \\
8\end{array}$ & & \begin{tabular}{l}
0 \\
8 \\
$\Xi$ \\
\hdashline \\
1
\end{tabular} & $\begin{array}{l}8 \\
\stackrel{8}{8} \\
\varnothing\end{array}$ & & m & 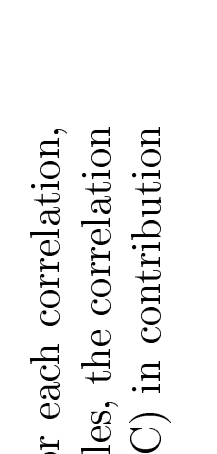 \\
\hline 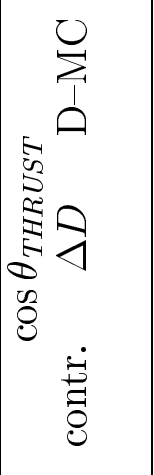 & & $\begin{array}{l}0 \\
8 \\
8 \\
0 \\
1\end{array}$ & $\begin{array}{l}\infty \\
0 \\
8 \\
8 \\
1 \\
1\end{array}$ & $\underset{1}{20}$ & $\begin{array}{l}12 \\
8 \\
8 \\
0\end{array}$ & 官 & 送 & ஓ & & 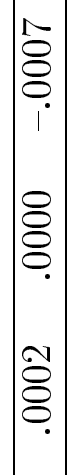 & $\begin{array}{l}10 \\
8 \\
8 \\
0\end{array}$ & ఠ & & $\stackrel{\Upsilon}{\mathscr{8}}$ & 8. & $\begin{array}{l}\mathscr{8} \\
\stackrel{8}{8}\end{array}$ & $\mid \begin{array}{l}m \\
8 \\
8 \\
0 \\
i\end{array}$ & 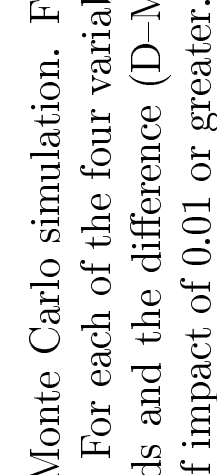 \\
\hline 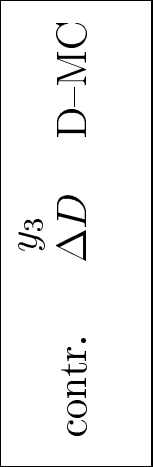 & 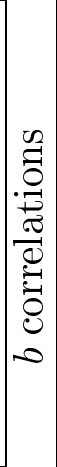 & 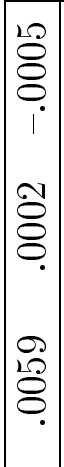 & $\begin{array}{l}1 \\
\mathscr{\infty} \\
\mathscr{8} \\
0\end{array}$ & 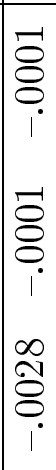 & $\begin{array}{l}\Omega \\
\stackrel{2}{2} \\
\stackrel{0}{0}\end{array}$ & 送 & 용 & 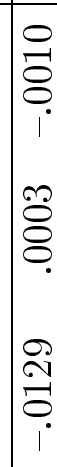 & 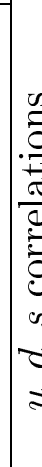 & 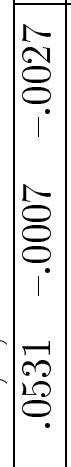 & 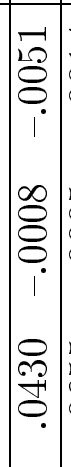 & $\begin{array}{l}10 \\
8 \\
8 \\
1 \\
1 \\
10 \\
10 \\
0 \\
0\end{array}$ & 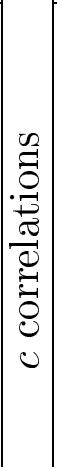 & 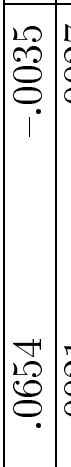 & 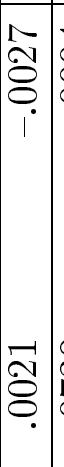 & $\begin{array}{l}\stackrel{+}{8} \\
8 \\
\\
\end{array}$ & @ & 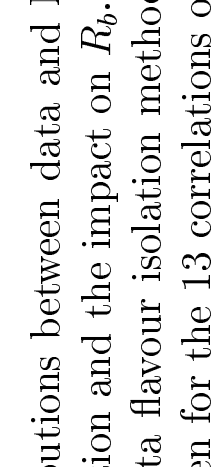 \\
\hline 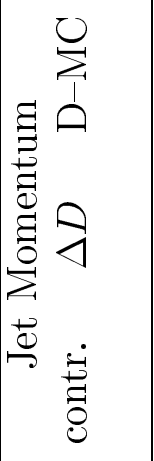 & & 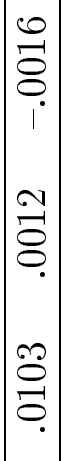 & ஓ्. & $\begin{array}{l}10 \\
8 \\
8 \\
0\end{array}$ & $\underset{\mathscr{F}}{\mathscr{\sigma}}$ & \& & 点 & 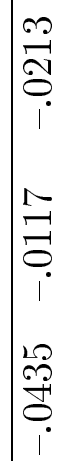 & & 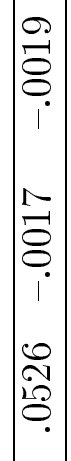 & 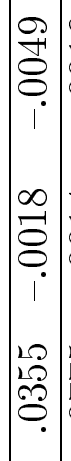 & 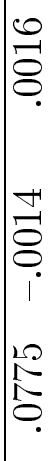 & & 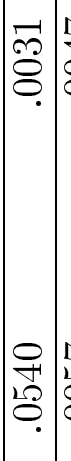 & $\stackrel{N}{\circledR}$ & 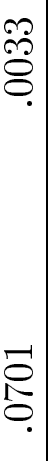 & 임 & 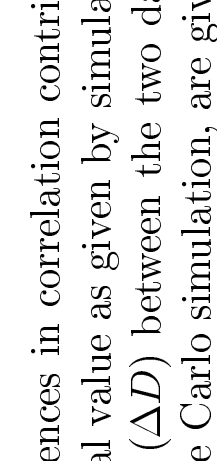 \\
\hline 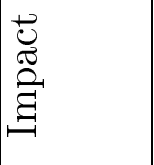 & & $\begin{array}{l}\stackrel{8}{8} \\
\stackrel{10}{7}\end{array}$ & $\underset{\sim}{\stackrel{\sim}{\sim}}$ & $\underset{\infty}{\infty}$ & $\underset{0}{\infty}$ & త્ & $\begin{array}{l}\stackrel{0}{0} \\
\stackrel{0}{0}\end{array}$ & $\bar{\delta}$ & & $\begin{array}{l}0 \\
0 \\
0 \\
1\end{array}$ & $\underset{1}{\stackrel{20}{+}}$ & $\begin{array}{c}0 \\
0 \\
0 \\
1\end{array}$ & & $\underset{1}{F}$ & $\vec{\partial}$ & $\stackrel{2}{-}$ & $\underset{\Xi}{g}$ & 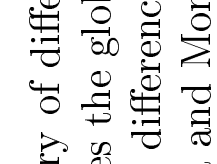 \\
\hline 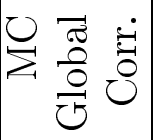 & & 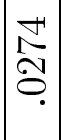 & 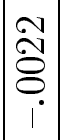 & 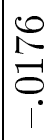 & $\begin{array}{l}0 \\
\stackrel{+}{1} \\
\stackrel{0}{0}\end{array}$ & 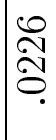 & $\begin{array}{l}8 \\
8 \\
8 \\
0\end{array}$ & $\begin{array}{l}0 \\
\stackrel{0}{8} \\
8\end{array}$ & & $\mid \begin{array}{l}-1 \\
0 \\
8 \\
0\end{array}$ & $\frac{\infty}{1}$ & 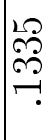 & & \begin{tabular}{l|l}
$\mathfrak{N}$ & 1 \\
1 & \\
$\infty$ & \\
0 &
\end{tabular} & $\mid \begin{array}{l}10 \\
\infty \\
8 \\
0 \\
0\end{array}$ & 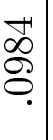 & $\frac{-00}{0}$ & 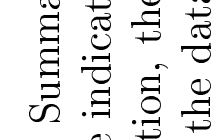 \\
\hline & & & & & & & & & & & & & & & & & 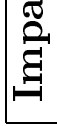 & 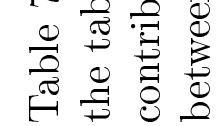 \\
\hline
\end{tabular}




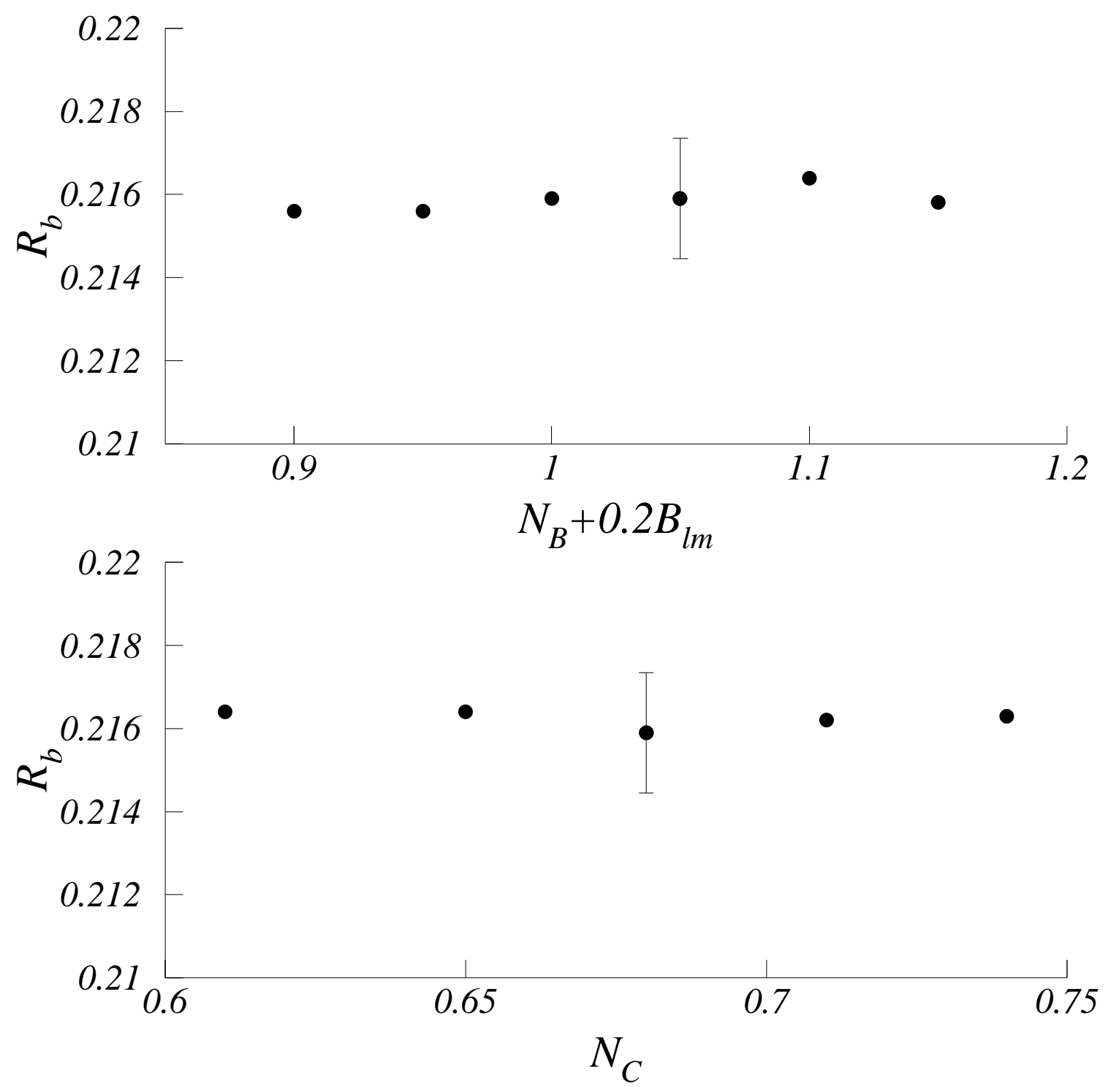

Figure 3: $R_{b}$ results as a function of the $\mathcal{N}_{B}$ cut of the $\mathrm{S}$ Tag (upper plot) and as a function of the $\mathcal{N}_{C}$ cut of the $\mathrm{C}$ Tag (lower plot). 


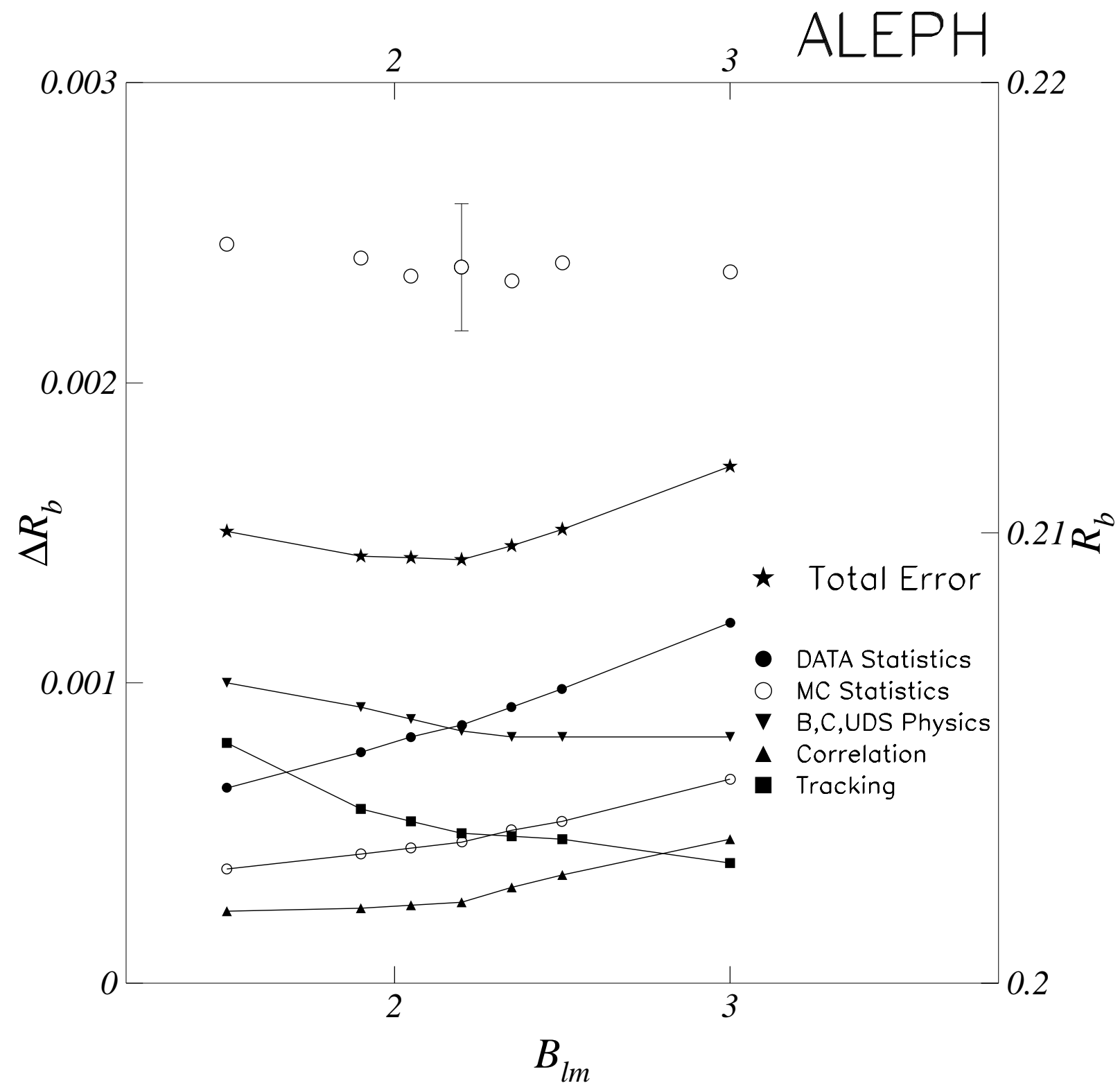

Figure 4: $R_{b}$ results as a function of the $\mathcal{B}_{l m}$ cut (upper plot) plotted against the right ordinate; the error bar is the total one. On the left ordinate the different sources of errors are plotted together with the total error as a function of the $\mathcal{B}_{l m}$ cut. 
If the radiative corrections, which are dominated by top quark effects, were left out of the electroweak calculation, the expected result would be:

$$
R_{b}=0.2183 \pm 0.0001 \quad \text { (Electroweak expectation without rad. corr.). }
$$

This measurement is an indication of the top quark dominated radiative vertex correction.

Other measurements of $R_{b}$ have been reported recently by SLD [9], DELPHI [10] and OPAL [11]. The more precise result, the one of OPAL, is $R_{b}=0.2175 \pm 0.0014$ (stat) \pm 0.0017 (syst) $-0.106 \times\left(R_{c}-0.172\right)$.

\section{Acknowledgements}

We are indebted to our colleagues in the accelerator divisions for the good performance of the LEP storage ring. We thank also the engineers and technicians of our home institutes for their support in constructing ALEPH. Those of us from non-member countries thank CERN for its hospitality. 


\section{Appendix}

\section{Neural network input variables for $\mathcal{N}_{B}$}

- $\mathrm{B}(1)$ : Longitudinal momentum $p_{\|}$of the leading track of the most energetic jet $J_{\text {max }}$ of the hemisphere.

- $\mathrm{B}(2)$ : Fox-Wolfram moment of order 5 normalized to the $0^{\text {th }}$ moment [12].

- $\mathrm{B}(3)$ : Transverse mass with respect to the plane of the event

$$
\mathrm{B}(3)=\frac{\sum_{i}\left|p(i) \cos \theta_{i 3}\right| \sqrt{s}}{E_{H e m i}}
$$

where the subscript 3 refers to the minor axis (axis perpendicular to the plane of the event), $i$ runs over all the energy flow tracks of the hemisphere (charged tracks, photons and neutral hadrons) and $E_{H e m i}$ is the visible energy of the hemisphere.

- $\mathrm{B}(4)$ : Invariant mass of the three most energetic tracks of $J_{\max }$.

- B(5) : "Forward Momentum" : tracks of the most energetic jet of the hemisphere are boosted along its axis $\vec{J}_{\text {max }}$ assuming that the B hadrons carry on average $70 \%$ of the beam energy, and the total momentum of the tracks produced in a forward direction with respect to $\vec{J}_{\text {max }}$ is computed.

- $\mathrm{B}(6)$ : Transverse momentum $p_{\perp}$ of the second leading track of $J_{\text {max }}$.

- $\mathrm{B}(7)$ : Same as B(6) for the leading track of $J_{\text {max }}$.

- $\mathrm{B}(8)$ : Multiplicity of the energy flow tracks of $J_{\max }$ with $p>1 \mathrm{GeV} / \mathrm{c}$.

- $\mathrm{B}(9)$ : Invariant mass of the charged tracks of $J_{\max }$ with $p>1 \mathrm{GeV} / \mathrm{c}$.

- $\mathrm{B}(10)$ : Invariant mass of the energy-flow tracks belonging to a cone of $\pm 40^{\circ}$ around the axis of $J_{\max }$ (denoted $C_{40}^{\text {Jet }}$ hereafter).

- B(11) : Sum of the squared transverse momenta of the particles belonging to the cone $C_{40}^{J e t}$.

- $\mathrm{B}(12)$ : Energy of $C_{40}^{\text {Jet }}$ divided by $E_{H e m i}$.

- B(13) : Energy of the leading track of the hemisphere divided by $E_{H e m i}$.

- $\mathrm{B}(14)$ : Invariant mass of the energy-flow tracks belonging to a cone of $\pm 40^{\circ}$ around the leading track of the hemisphere (denoted $C_{40}^{\text {track }}$ hereafter).

- B(15) : Energy of $C_{40}^{\text {track }}$ divided by $E_{\text {Hemi }}$.

- $\mathrm{B}(16)$ : "Directed Sphericity" [13] defined as : $\frac{\sum_{i} p_{\perp}(i)^{2}}{\sum_{i}|P(i)|^{2}}$, where $i$ runs over a set of energy flow tracks of $J_{\max }, p_{\perp}(i)$ is the transverse momentum of the track $i$ w.r.t. its jet axis and $P(i)$ is the momentum of $i$ estimated in the centre of mass of the set of considered tracks. This variable is an attempt to reflect the fact that the decay of a $B$-hadron in its rest frame is more isotropic than for light hadrons. Here the set of tracks used is the first, second and third most energetic energy flow tracks of $J_{\max }$.

- B(17) : Same as B(16) for the first, second and fourth tracks of $J_{\max }$.

- B(18) : Same as B(16) for the first and third tracks of $J_{\max }$.

- B(19) : Same as B(16) for the first and fourth tracks of $J_{\max }$. 
- B(20) : Same as B(4) for the first and third tracks of $J_{\max }$.

- B(21) : Energy (in the laboratory frame) of the set of tracks used in B(16).

- B(22) : Energy of the set of tracks used in B(17).

- B(23) : Energy of the first and second most energetic tracks of $J_{\max }$.

- B(24) : Energy of the set of tracks used in B(19).

- $\mathrm{B}(25)$ : Energy of the system of tracks obtained with a nucleated jet algorithm starting from the most energetic track of the hemisphere and stopping the nucleation when the invariant mass exceeds $2.1 \mathrm{GeV} / \mathrm{c}^{2}$ [14]. This variable is intended to reproduce in an inclusive way the $\left\langle X_{E}\right\rangle$ of $D$ mesons (which is different for $D$ mesons produced in $Z \rightarrow c \bar{c}$ and $Z \rightarrow b \bar{b}$ events).

\section{Neural network input variables for $\mathcal{N}_{C}$}

- $\mathrm{C}(1)$ : This variable is defined as :

$$
C(1)=\frac{\sum_{j}\left[\sum_{i \in j} p_{\perp}(i)\right]}{P_{H e m i}}
$$

where $j$ runs over all the jets of the hemispheres.

- $\mathrm{C}(2)$ : Same as B(11) for the tracks of $J_{\text {max }}$.

- $\mathrm{C}(3)$ : This variable is defined as :

$$
C(3)=\frac{\sum_{j}\left[\sum_{i} p_{\perp}(i) p_{\|}(i)\right]}{P_{\text {Hemi }}^{2}}
$$

where $j$ runs over all jets of the hemisphere and $i$ over all the tracks of each jet $j$.

- $\mathrm{C}(4)$ : Same as B(1).

- $\mathrm{C}(5)$ : Longitudinal momentum $p_{\|}$of the second leading track of $J_{\text {max }}$.

- $\mathrm{C}(6)$ : Same as B(7).

- $\mathrm{C}(7)$ : Same as B(3).

- $\mathrm{C}(8)$ : Visible energy of the hemisphere $E_{\text {Hemi }}$.

- $\mathrm{C}(9)$ : "Directed Sphericity" calculated with the four most energetic tracks of $J_{\max }$.

- C(10) : Longitudinal momentum $p_{\|}$of the third leading track of $J_{\max }$.

- $\mathrm{C}(11)$ : Same as B(5).

- $\mathrm{C}(12)$ : Invariant mass of $J_{\max }$.

- $\mathrm{C}(13)$ : Sum of the masses of all the jets of the hemisphere.

- $\mathrm{C}(14): \mathcal{P}_{H}$ as described in the text.

- $\mathrm{C}(15)$ : Multiplicity of the charged tracks of $J_{\max }$ with $p>0.25 \mathrm{GeV} / \mathrm{c}$.

- C(16) : Multiplicity of the charged tracks of $J_{\max }$ with $p>1.0 \mathrm{GeV} / \mathrm{c}$.

- $\mathrm{C}(17)$ : Multiplicity of the energy flow tracks of $J_{\max }$ with $p>0.25 \mathrm{GeV} / \mathrm{c}$.

- $\mathrm{C}(18)$ : Same as B(8).

- $\mathrm{C}(19)$ : Invariant mass of the energy flow tracks of $J_{\max }$ with $p>1 \mathrm{GeV} / \mathrm{c}$.

- $\mathrm{C}(20)$ : Number of identified leptons (electrons and muons) with $p>3 \mathrm{GeV} / \mathrm{c}$ in the hemisphere. 


\section{References}

[1] D. Buskulic et al., (ALEPH Collab.), A Measurement of $R_{b}$ using a LifetimeMass Tag, CERN PPE/97-017. To be submitted to Physics Letters B.

[2] D. Buskulic et al., (ALEPH Collab.), Nucl. Instr. Meth.A360 (1995) 481.

[3] W. Bartel et al., (JADE Collab.), Z. Phys. C33 (1986) 23.

[4] D. Buskulic et al., (ALEPH Collab.). Phys. Lett. B313 (1993) 549.

[5] D. Buskulic et al., (ALEPH Collab.). Nucl. Instr. Meth. A 346 (1995) 461.

[6] Presentation of LEP Electroweak Heavy Flavour Results for Summer 1996 Conferences, LEPHF/96-01, ALEPH note 96-099.

[7] Reports of the Working group on Precision Calculations for the $Z$ resonance, Eds. D.Bardin, W.Hollik and G.Passarino, CERN 95-03 and references therein.

[8] J .Lys, (CDF Collab.), FERMILAB-CONF-96/409-E. Proceedings ICHEP'96, Warsaw,(1996); E. W. Warnes, (D0 Collab.), FERMILAB-CONF-96/243-E, Proceedings ICHEP'96, Warsaw,(1996); M. Demarteau, FERMILAB-CONF96/354, Proceedings DPF'96, Minneapolis,(1996).

[9] K. Abe et al., (SLD Collab.), Phys. Rev. D53 (1996) 1023.

[10] P. Abreu et al., (DELPHI Collab.), Z. Phys. C70 (1996) 531.

[11] K. Ackerstaff et al., (OPAL Collab.), CERN-PPE-96-167 (1996), (submitted to Z. Phys. C).

[12] G. C. Fox, S. Wolfram, Phys. Rev. Lett., 41 (1978) 1581.

[13] L. Bellantoni et al., Nucl. Instr. Meth. A310 (1991) 618.

[14] D. Buskulic et al., (ALEPH Collab.), Phys. Lett. B295 (1992) 396. 